\title{
LOCAL CONTRACTIBILITY, CELL-LIKE MAPS, AND DIMENSION
}

\author{
JAN van MILL ${ }^{1}$
}

\begin{abstract}
We consider the existence of cell-like maps $f: I^{n} \rightarrow X$ such that no nonempty open subset of $X$ is contractible in $X$. From the Taylor Example, it is easy to construct such a map for $n=\infty$. We show that there exists such a map for some finite $n$ if (and only if) there exists a dimension raising cell-like map of a compactum.
\end{abstract}

1. Introduction. All spaces are separable metric. A compactum $X$ has trivial shape if every continuous function from $X$ to a polyhedron is null homotopic. In addition, a continuous surjection $f: X \rightarrow Y$ between compact spaces is called cell-like provided that $f^{-1}(y)$ has trivial shape for every $y \in Y$. One of the most outstanding open problems in geometric topology is whether a cell-like map of a compactum can raise dimension. This problem is known as the "cell-like dimension raising mapping problem" and has many equivalent formulations. For example, the cell-like dimension raising mapping problem is equivalent to the problem whether every compactum with finite cohomological dimension is finite dimensional (Edwards; for details, see Walsh [8]) and also to the problem whether the cell-like image of a (finite-dimensional) compact manifold is an ANR (Kozlowski [6]; see also Ancel [1]). Since every finite-dimensional space imbeds in $I^{n}$ for some $n<\infty$ (here $I$ denotes the interval $[0,1]),[4,1.11 .4]$, several problems related to cell-like maps are in fact problems concerning cell-like maps defined on the $n$-cubes $I^{n}, n<\infty$.

In [1], Ancel asked whether the cell-like image of a compact ANR is locally contractible. It is known that such an image is $\mathrm{LC}^{\infty},[2,12.1]$, but need not be an ANR, [7]. Relevant to Ancel's question is also that Daverman and Walsh [3] used the Taylor Example [7] to produce a cell-like map $f: I^{\infty} \rightarrow X$ such that $X$ is not an ANR but is locally contractible.

We shall establish the following results.

THEOREM. If for every cell-like map $f: I^{n} \rightarrow X, n<\infty$, there exists a nonempty open subset of $X$ which is contractible in $X$, then no cell-like map of a compactum raises dimension (and conversely).

Received by the editors September 20, 1985 and, in revised form, October 28, 1985.

1980 Mathematics Subject Classification (1985 Revision). Primary 55M10, 54C35.

Key words and phrases. Cell-like map, dimension, local contractibility.

${ }^{1}$ This note was written while the author was visiting the Department of Mathematics at Wesleyan University in August 1985. The author is pleased to thank Wesleyan University for generous hospitality and support. 
EXAMPLE. There exists a cell-like image of the Hilbert cube such that no nonempty open subset is contractible in the space.

I am indebted to Doug Curtis for some helpful comments.

2. Proofs. The proof of our theorem is in two steps.

2.1. Lemma. Let $n \in \mathbf{N} \cup\{\infty\}$. If for every cell-like map $f: I^{n+1} \rightarrow X$, there exists a nonempty open subset of $X$ which is contractible in $X$, then every cell-like image of $I^{n}$ is contractible.

Proof. Let $f: I^{n} \rightarrow Y$ be cell-like. In addition, let $\left\{X_{i}: i \in \mathbf{N}\right\}$ be a null sequence of disjoint compacta in $I^{n+1}$ such that

(1) $X_{i}$ is homeomorphic to $I^{n}$ for every $i$;

(2) if $U$ in $I^{n+1}$ is open and nonempty then there is an $i \in \mathbf{N}$ with $X_{i} \subseteq U$.

(It is of course a triviality to construct such a sequence.) Let $\left\{Y_{i}: i \in \mathbf{N}\right\}$ be a sequence of disjoint copies of $Y$ and for each $i \in \mathbf{N}$ let $f_{i}: X_{i} \rightarrow Y_{i}$ be a copy of the map $f$, i.e. $f_{i}$ has the form $\eta \circ f \circ \xi^{-1}$ for certain homeomorphisms $\xi: I^{n} \rightarrow X_{i}$ and $\eta: Y \rightarrow Y_{i}$. It is easy to prove (and well known) that the collection

$$
\mathscr{G}=\left\{f_{i}^{-1}(y): y \in Y_{i}, i \in \mathbf{N}\right\} \cup\left\{\{x\}: x \in I^{n+1} \backslash \bigcup_{i=1}^{\infty} X_{i}\right\}
$$

is an upper-semicontinuous decomposition of $I^{n+1}$. Put $Z=I^{n+1} / \mathscr{G}$ and let $\pi$ : $I^{n+1} \rightarrow Z$ be the corresponding quotient map. For convenience we shall identify $\pi\left(X_{i}\right)$ and $Y_{i}$ for every $i$. Clearly, $\pi$ is cell-like. By assumption, there exists an open set $U$ in $Z$ which is contractible in $Z$ and nonempty. Since $\pi^{-1}(U)$ is open in $I^{n+1}$ and nonempty, by (2) there exists an $i \in \mathbf{N}$ with $X_{i} \subseteq \pi^{-1}(U)$. Consequently, $Y_{i}=\pi\left(X_{i}\right) \subseteq U$ from which follows that $Y_{i}$ is contractible in $Z$. We shall prove that $Y_{i}$ is also a retract of $Z$. It then follows that $Y_{i}$ is contractible and since $Y_{i}$ is homeomorphic to $Y$ we conclude that $Y$ is contractible, as required. Let $\mathscr{H}$ be the upper-semicontinuous decomposition of $I^{n+1}$ with nondegenerate elements $\left\{f_{j}^{-1}(y)\right.$ : $\left.y \in Y_{j}, j \in \mathbf{N} \backslash\{i\}\right\}$ and let $T=I^{n+1} / \mathscr{H}$. The corresponding quotient map from $I^{n+1}$ shall be denoted by $\theta$. Clearly, $X_{i}$ and $\theta\left(X_{i}\right)$ are homeomorphic from which follows, by (2), that there is a retraction $r: T \rightarrow \theta\left(X_{i}\right)$. There clearly exists a continuous function $\xi: T \rightarrow Z$ such that

(3) $\xi\left(\theta\left(X_{i}\right)\right)=Y_{i}$,

(4) $\xi$ restricted to $T \backslash \theta\left(X_{i}\right)$ is a homeomorphism from $T \backslash \theta\left(X_{i}\right)$ onto $Z \backslash Y_{i}$. Now define $s: Z \rightarrow Y_{i}$ by

$$
s(y)= \begin{cases}y & \left(y \in Y_{i}\right), \\ \xi r \xi^{-1}(y) & \left(y \in Z \backslash Y_{i}\right) .\end{cases}
$$

An easy check shows that $s$ is a retraction.

2.2. Lemma. Let $n \in \mathbf{N} \cup\{\infty\}$. If every cell-like image of $I^{2 n+1}$ is contractible then no cell-like map of an at most $n$-dimensional compactum raises dimension.

Proof. Let $X$ be a compact space with $\operatorname{dim} X \leqslant n$ and let $f: X \rightarrow Y$ be cell-like. If $n=\infty$ then there is nothing to prove, so assume that $n<\infty$. We shall prove that $\operatorname{dim} Y \leqslant 2 n+1$ from which follows that $\operatorname{dim} Y \leqslant \operatorname{dim} X$ since the Vietoris Theorem 
implies that the cohomological dimension of $Y$ is less than or equal to $\operatorname{dim} X$. By [4, 1.11.4] we may assume that $X \subseteq I^{2 n+1}$. Let $A \subseteq Y$ be closed. Put $B=f^{-1}(A)$ and let $g=f \mid B$. Let $\xi: A \rightarrow S^{2 n+1}$ be continuous. We shall prove that $\xi$ is null homotopic. To this end, let $Z=I^{2 n+1} \cup_{g} A$. By assumption, $Z$ is contractible. Also, observe that $\operatorname{dim} Z \backslash A \leqslant 2 n+1$. Consequently, the function $\xi$ can be extended to a continuous function $\eta: Z \rightarrow S^{2 n+1}[4,1.9 .2]$. Since $Z$ is contractible, $\eta$ is null homotopic, so $\xi$ is null homotopic.

By the Borsuk Homotopy Extension Theorem [4, 1.9.7], we can now extend $\xi$ to a continuous function $\bar{\xi}: Y \rightarrow S^{2 n+1}$. Since $A$ was arbitrarily chosen, it now follows from $[4,1.9 .3]$ that $\operatorname{dim} Y \leqslant 2 n+1$, as required.

It is easily seen that by Lemmas 2.1 and 2.2 the proof of our theorem is completed.

We shall now construct the required example. Keesling [5] used the Taylor Example [7] to get a cell-like map $f: I^{\infty} \rightarrow X$ such that $X$ is not movable. In particular, $X$ cannot have the shape of an AR and is therefore not contractible. By Lemma 2.1 there consequently exists a cell-like image of $I^{\infty}$ such that no nonempty open subset is contractible in the space. Alternatively, one can get such an example via the following procedure. For any space $Y$ let $Y^{\infty}$ denote the countable infinite product of copies of $Y$. Let $f$ and $X$ be such as above. Define $F:\left(I^{\infty}\right)^{\infty} \rightarrow X^{\infty}$ by $F=f \times f \times f \times \cdots$. Then $F$ is clearly cell-like and no nonempty open subset of $X^{\infty}$ is contractible in $X^{\infty}$ since $X$ is not contractible.

ADDED IN PROOF. J. Mogilski kindly informed me that Lemma 2.2 was also obtained independently by S. Nowak, Some extension and classification theorems of movable spaces, to appear in Fund. Math.

\section{REFERENCES}

1. F. D. Ancel, The role of countable dimensionality in the theory of cell-like relations, Trans. Amer. Math. Soc. 287 (1985), 1-40.

2. K. Borsuk, Theory of retracts, PWN, Warsaw, 1967.

3. R. J. Daverman and J. J. Walsh, Example of cell-like maps that are not shape equivalences, Michigan Math. J. 30 (1983), 17-30.

4. R. Engelking, Dimension theory, PWN, Warsaw, 1978.

5. J. Keesling, A non-movable trivial-shape decomposition of the Hilbert cube, Bull. Acad. Polon. Sci. 23 (1975), 997-998.

6. G. Kozlowski, Images of $A N R$ 's, unpublished manuscript.

7. J. L. Taylor, A counterexample in shape theory, Bull. Amer. Math. Soc. 81 (1975), 629-632.

8. J. J. Walsh, Dimension, cohomological dimension, and cell-like mappings, Shape Theory and Geometric Topology, Lecture Notes in Math., vol. 870, Springer-Verlag, Berlin and New York, 1981, pp. 105-118.

Subfaculteit Wiskunde, Vrije Universiteit, De BoelelaAn 1081, 1081 HV Amisterdam, The NETHERLANDS

Mathematisch Institudt, Universiteit van Amsterdam, RoetersstraAt 15, Amsterdam, The NetherLANDS 